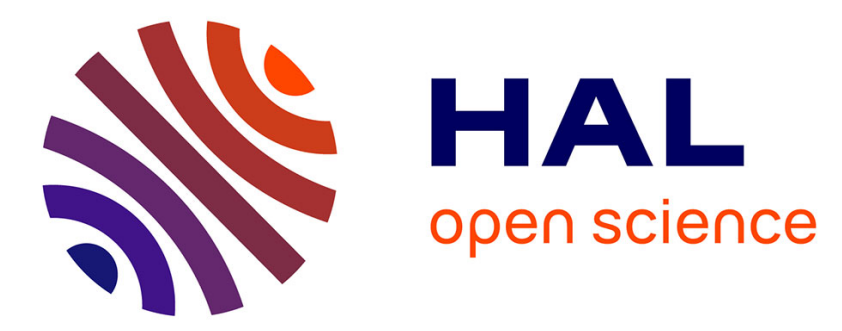

\title{
Surface viscoelasticity in model polymer multilayers: From planar interfaces to rising bubbles
}

M. Pepicelli, N. Jaensson, C. Trégouët, B. Schroyen, A. Alicke, T. Tervoort, C. Monteux, J. Vermant

\section{- To cite this version:}

M. Pepicelli, N. Jaensson, C. Trégouët, B. Schroyen, A. Alicke, et al.. Surface viscoelasticity in model polymer multilayers: From planar interfaces to rising bubbles. Journal of Rheology, 2019, 63 (5), pp.815-828. 10.1122/1.5096887. hal-02321073

\section{HAL Id: hal-02321073 \\ https://hal.science/hal-02321073}

Submitted on 20 Oct 2019

HAL is a multi-disciplinary open access archive for the deposit and dissemination of scientific research documents, whether they are published or not. The documents may come from teaching and research institutions in France or abroad, or from public or private research centers.
L'archive ouverte pluridisciplinaire HAL, est destinée au dépôt et à la diffusion de documents scientifiques de niveau recherche, publiés ou non, émanant des établissements d'enseignement et de recherche français ou étrangers, des laboratoires publics ou privés. 


\title{
Surface viscoelasticity in model polymer multilayers: from planar interfaces to rising bubbles
}

\author{
M. Pepicelli, ${ }^{1, *}$ N. Jaensson, ${ }^{1, *}$ C. Tregouët, ${ }^{2}$ B. Schroyen, ${ }^{1}$ A. Alicke, ${ }^{1}$ T. Tervoort, ${ }^{1}$ \\ C. Monteux, ${ }^{2, \dagger}$ and J. Vermant ${ }^{1, \dagger}$ \\ ${ }^{1}$ Department of Materials ETH Zurich Vladimir-Prelog-Weg 5 Zurich 8093 Switzerland \\ ${ }^{2}$ Laboratoire Sciences et Ingénierie de la Matière Molle PSL Research University UPMC \\ Univ Paris 06 ESPCI Paris UMR 7615 CNRS 10 rue Vauquelin 75231 Paris Cedex 05 France
}

(Dated: July 19, 2019)

\begin{abstract}
In the present work a polymeric transient viscoelastic network is used as a model system to investigate several fundamentals of interfacial viscoelasticity and non-linear behavior, in simple shear, compression and for simple mixed deformations. A supramolecular polymer bilayer, characterized by long but finite relaxation times, is created at the water-air interface using a layer-by-layer assembly method. The possibility of studying the individual layers starting from an unstrained reference state enabled the independent quantification of the equilibrium thermodynamic properties, and the viscoelastic response of the bilayer could be studied separately for shear and compressional deformations. Time- and frequency-dependent material functions of the layer were determined in simple shear and uniform compression. Moreover, a quasi linear neo-Hookean model for elastic interfaces was adapted to describe step strain experiments on a viscoelastic system by allowing the material properties to be time-dependent. The use of this model made it possible to calculate the response of the system to step deformations. Within the linear response regime, both stress-strain proportionality and the superposition principle were investigated. Furthermore, the onset of non-linear behavior of the extra stresses was characterized in shear and for the first time in pure compression. We conclude by investigating the multilayer system in a rising bubble setup and show that the neo-Hookean model is able to predict the extra and deviatoric surface stresses well, up to moderate deformations.
\end{abstract}

\section{INTRODUCTION}

Interfacial rheology plays a crucial role in so-called highinterface materials, which are found in many industrial and biological applications, ranging from foods and pharmaceutics to those using chemical processes. For example, polymer capsules, which are used as targeted drugs delivery systems, rely on interfacial mechanics, lung surfactant replacements can be improved by considering their surface properties, and production processes of multiphase products benefit from increased knowledge of interfacial rheology [1-6].

As in bulk, the proposed approach of interfacial rheology is to study the response of complex fluid-fluid interfaces in kinematically simple deformations in order to obtain material functions. These functions can then be used to parametrize constitutive equations to predict the response of the interfaces and bulk fluids for more complex situations. However, additional mechanisms come into play once an interface is deformed, which can be of different nature. In general, changes in the equilibrium thermodynamic properties and phenomena related to bulk and interfacial transport phenomena, are coupled with rheological contributions $[5,7,8]$. For this reason, a correct quantification of the rheological properties of interest can only be achieved if each contribution is well identified and elucidated. Key to this is the application of well-defined

\footnotetext{
* These two authors contributed equally

$\dagger$ Cecile.Monteux@espci.fr

† Jan.Vermant@mat.ethz.ch
}

deformations and the consequent correct determination of the different material functions in these varying standard flows. Moreover, due to the inherent coupling between the interface and the subphase(s), these material functions may only be defined with respect to a certain subphase for surfaces, or a combination of subphases for interfaces.

For deformations of the interface at constant area, the level of accuracy and sensitivity reached by modern interfacial rheometrical techniques is high, especially in shear [9-13]. For deformations of the interface at changing area, i.e. dilatational/compressional rheology, the situation is not as clearcut. The flow field in the devices that are currently available is often mixed and the analysis involves different contributions that need to be accounted for, especially in the presence of structured interfaces with extra, possibly deviatoric, stresses [14-16]. Recently, it has been shown how to correctly determine pure dilatational/compressional moduli as a function of applied strain using a radial trough geometry [15]. Moreover, a complete and self-consistent experimental analysis of an elastic interface allowed the validation of a material-frame indifferent neo-Hookean model that describes large elastic deformations of interfaces with finite elasticity. For further reading on interfacial rheology for complex interfaces, the reader is referred to the recent review of Jaensson and Vermant [17].

When studying these fundamental rheological properties, choosing an appropriate model system is not obvious. Soluble small molecular weight surfactant molecules crowd the interface but are claimed to have immeasurably small shear viscosities [18], fatty acids such as eicosanol or hexadecanol show a predominantly viscous response in shear and even dilation [19, 20] and phospholipids such as Dipalmitoylphos- 
phatidylcholin (DPPC) can be strongly viscoelastic below its melting temperature, but with strong dependencies on the deformation and thermal histories [21-23]. Colloidal systems, either stable or aggregated, show significant storage moduli as surface coverage is increased [24-27], but the behavior is typically visco-plastic, i.e. they exhibit a yield stress. Some proteins (such as hydrophobin class II) display significant viscoelastic responses, but are somewhat challenging to manipulate with very long relaxation times $[28,29]$. To come to model viscoelastic interfaces, one needs a system where the rheological properties are sufficiently strong, but where the structures can be assembled without generating an initial, unknown, pre-strained state due to a pre-compression or to the interface formation itself.

Certain polymeric systems are capable of forming transient networks which fulfill the requirements for such a model system. It has been shown that the properties of hydrogen bonded polymer multilayers can be tuned. For example, polymer multilayers made from Polyvinylpyrrolidone (PVP) as a hydrogen-acceptor and hydrogen donors such as poly(methacrylic acid) (PMAA) or poly(acrylic acid) (PAA) have been shown to have interfacial rheological properties which can be tuned by changing the $\mathrm{pH}$, the hydrophobic interactions or even by anchoring the polymers to the interface using grafted alkane moieties $[6,30]$. Whereas the individual polymers, which are adsorbed in a configuration consisting of "trains and loops", are very compressible as there is a fast exchange between loops and trains, polymer bilayers, with hydrophobic interactions and hydrogen bonding, form a strong polymer network where this is no longer the case. More elastic networks are formed between PMAA and PVP compared to PAA/PVP because of stronger hydrophobic interactions. Hydrophobic anchors can be further introduced to control the interactions [30]. In the present work we use a PVP/PMAA bilayer, schematically shown in Figure 1, as a model transient network layer.

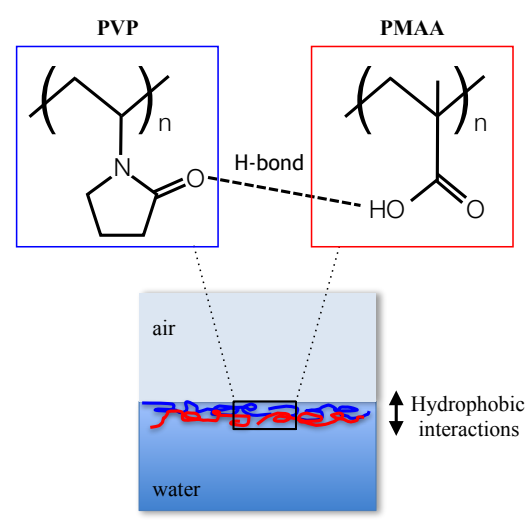

FIG. 1. Chemical structure of PVP and PMAA (top) and schematic showing the origin of the interlayer interactions (bottom) with the role of hydrogen bonding and hydrophobic interactions.

These bilayers can be assembled by a Layer-by-Layer $(\mathrm{LbL})$ assembly method at planar interfaces using optimized subphase exchange cells for both simple shear rheometry and pure dilatational/compressional rheometrical setups. We find this PVP/PMAA system to exhibit long but finite relaxation times, which, combined with the layer-by-layer assembly, enables us to generate interfaces which are initially unstrained, and the material functions at an aqueous subphase can be measured for well-defined shear and compressional deformations. At the same time, we can deform the bilayer at high Deborah numbers so that the elastic response in step strain experiments can be quantified (in shear and compression) with a quasilinear viscoelastic model. The goal of the present work is to obtain a full characterization of the rheological response of this model viscoelastic interface, to validate recently proposed quasi linear constitutive equations and aspects such as Boltzmann superposition principle for the extra stresses, in both shear and compression. The transient networks studied here are well suited due to the network nature of he material, and a significant values for the linear viscoelastic interfacial moduli have been reported $[6,30]$, and depending on the strength of the interactions these can be well separated from the changes in thermodynamic properties, which is more difficult for other types of interfaces such as simple polymer layers. Using the experimentally determined materials functions and the model, the anisotropic stress state for the more complex deformation occurring for a rising bubble, where the interface is subjected to both shear and compressional strains, is predicted and compared to experimental observations.

\section{MATERIALS AND METHODS}

\section{A. Sample preparation}

Solutions of PMAA (Polysciences Inc, molecular weight $\mathrm{M}_{w}=100$ '000 $\mathrm{g} / \mathrm{mol}$ ) and PVP (Sigma-Aldrich, $\mathrm{M}_{w}=30$ '000 $\mathrm{g} / \mathrm{mol}$ ) were prepared by dissolving each polymer in deionized water (Millipore Milli-Q system, resistivity $18.2 \mathrm{M} \Omega \mathrm{cm}$ ) at a concentration of $1 \mathrm{wt} \%$ and by gently stirring until complete dissolution. The $\mathrm{pH}$ of both solutions was then adjusted to 3 using a 1 molar solution of hydrochloric acid $(\mathrm{HCl})$ and a 1 molar solution of sodium hydroxide $(\mathrm{NaOH})$. In the present work, the multilayers are assembled at the air-water interface using a layer-by-layer (LbL) method $[2,3,6]$. The polymeric solutions are introduced in the experimental apparatus sequentially, allowing in each step the adsorption of a single polymer layer and subsequently removing the bulk phases by injecting acidic water. The acidic solutions of Milli-Q water were prepared at $\mathrm{pH} 3$ using a 1 molar solution of $\mathrm{HCl}$. It was shown that a $\mathrm{pH}$ value of 3 represents the optimal experimental condition for maximising both hydrogen bonds and hydrophobic interactions [6]. In order to avoid polymer degradation, the solutions were stored at $4{ }^{\circ} \mathrm{C}$ and kept for no longer than a week after preparation. Prior to the start of each measurement, the solutions were allowed to equilibrate at room temperature and stirred for 1 hour. 


\section{B. Interfacial compressional rheology}

The compressional properties of PVP/PMAA bilayers at an air-water interface were studied in a custom-made radial trough using linear compressions, as well as in oscillatory straining mode. Compared to the standard Langmuir trough, the radial symmetry creates a uniform area change without the influence of shear effects, as the shape of the interface is preserved during the experiments. This deformation is applied by an elastic band held in place by twelve aluminium fingers able to move simultaneously, controlled by a single stepper motor. A technical description of the setup is presented elsewhere [15].

The response of the interface was interrogated by either continuous compressions or by applying small amplitude area oscillations. In the latter case, sinusoidal area changes of $\pm 1 \%$ area strain amplitude were applied in a frequency range between 0.1 and $1 \mathrm{rad} / \mathrm{s}$. Frequency sweeps were performed at surface pressures of 20,30 , and $38 \mathrm{mN} / \mathrm{m}$. In order to reach these pre-defined pressure values, the interface was linearly compressed at a speed of $1.5 \mathrm{~mm} / \mathrm{min}$. After each compression, the surface pressure was allowed to relax to an equilibrium value prior to execution of the oscillatory measurement. Strain amplitude sweeps were carried out on an initially unstrained interface directly after assembly, and at the end of the series of measurements, ranging the area change from $0.2 \%$ to $8 \%$ at a frequency of $0.25 \mathrm{rad} / \mathrm{s}$. The results indicated that the strain amplitude of $1 \%$ area change is in the linear viscoelastic regime (data not shown).

Surface pressures were recorded using a Wilhelmy balance (KSV-NIMA, Finland) equipped with a rod of $1.05 \mathrm{~mm}$ diameter (KSV-NIMA, Finland) to keep the radial symmetry in the radial trough. All measurements were carried out at controlled room temperature $\left(22 \pm 1{ }^{\circ} \mathrm{C}\right)$.

\section{Interfacial shear rheology}

The shear rheological properties of the PVP/PMAA bilayer were determined using a stress controlled DHR-3 rheometer (TA instruments, USA) equipped with a modified Double Wall Ring (DWR) cup geometry that allows for the exchange of the subphase [31]. In order to guarantee the same initial conditions, a time sweep $(0.05 \%$ strain amplitude at $1 \mathrm{rad} / \mathrm{s}$ ) was performed (data not shown) at the beginning of each experiment to assess that the interface was fully equilibrated. Only then, the oscillatory experiments were started. Frequency sweeps were measured at a strain amplitude of $0.1 \%$. The extent of the linear viscoelastic regime was determined by an amplitude sweep, carried out at a frequency of $0.25 \mathrm{rad} / \mathrm{s}$. To ensure a negligible contribution of subphase drag to the total stress measured, the flow curve of the bulk solution was separately measured using a stress controlled MCR302 rheometer (Anton Paar, Austria), showing a mainly Newtonian behavior. The Boussinesq number for this system was calculated to be $B o=\left|\eta_{\mathrm{s}}^{*}\right| /(\eta L)>10^{4}$, where $\left|\eta_{\mathrm{s}}^{*}\right|$ is the experimentally measured magnitude of the complex surface shear viscosity, $\eta$ is the viscosity of the subphase and $L$ is a geometrical factor [11], implying that subphase contributions to the measured mechanical response are negligible. All measurements were performed at controlled room temperature $\left(22 \pm 1{ }^{\circ} \mathrm{C}\right)$.

\section{Rising bubble experiments}

The PVP/PMAA bilayer at an air-water interface was also investigated in a rising bubble experiment. A schematic of the setup that was used to generate and analyse the bubble is shown in Figure 2A, together with the solvent exchange cell used to created the LbL bilayer structure. After assembling the bilayer at the air-water interface, compression experiments were performed by decreasing the volume of the bubble.

The pressure inside the bubble was monitored directly using a pressure sensor (Omega PXM409-025HCGUSBH), which allowed for the measurement of the pressure with an accuracy of $\pm 1 \mathrm{~Pa}$. In order to calculate the pressure jump across the interface, the hydrostatic contribution to the pressure measurement must be known, i.e. the height of liquid above the capillary given by $h$ in Figure 2A. We determined $h$ by using a PTFE-coated needle on a micrometer that can move in the vertical direction. Before the experiment, the needle was lowered until it touched the capillary tip, and was subsequently moved up, out of the container. During the experiments, the needle was lowered until it touched the free surface of the liquid in the container, which was clearly visible by the induced deformation. The height of liquid, following from the difference between the two measurements, could be determined up to an accuracy of $100 \mu \mathrm{m}$, which corresponds to an accuracy in the hydrostatic pressure of $\pm 1 \mathrm{~Pa}$. This procedure is similar to the procedure presented in [32], with the exception that in the present work, the height was measured directly with the micrometer, circumventing an image-analysis step that could introduce additional errors in the measurement. The height between the needle tip and the pressure sensor also adds a small hydrostatic contribution to the measured pressure, but due to the low density of air, it sufficed to measure this height by hand for the given accuracy of $\pm 1 \mathrm{~Pa}$. The volume of the bubble was controlled by performing a real-time image analysis to obtain the bubble volume, and using a MATLAB script to control a Harvard syringe pump interfacing with an Arduino UNO R3 micro controller.

The bubble was illuminated using a green LED in front of a diffuser, and images were acquired using a camera from PointGrey (Grashopper3), through a telecentric lens from Stemmer Imaging (SILL TZM 4425/1,0-C). The contour of the bubble was detected as a combination of $r$ and $z$ coordinates. Following the approach as presented by Nagel et al. [16], the interface coordinates are then projected on Chebyshev basis functions of the first kind, yielding an accurate numerical description of the interface, as shown in Figure 2B. By minimizing the error between a shape that satisfied the Young-Laplace equation and the experimental shape, the unknown surface tension and pressure were then calculated and compared to the measured pressure. The code used in the rising bubble experiments is based on the code published before by Nagel et 
al. [16], which is available under an open-source license.

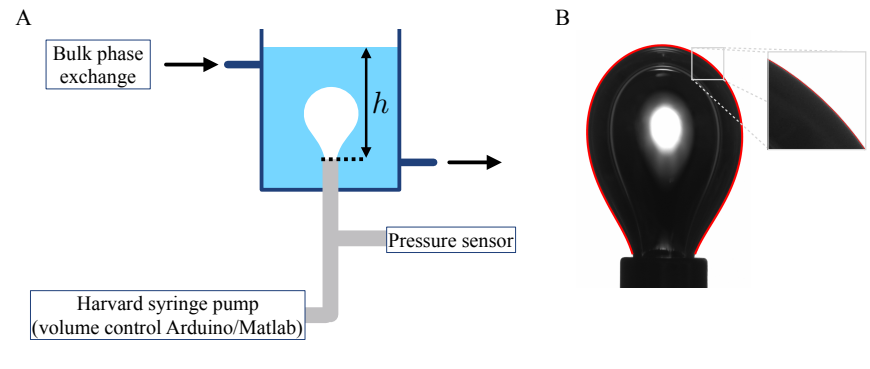

FIG. 2. Schematic overview of the rising bubble experiment (A). An example of an image of a rising bubble, with the bubble surface as found by the image processing step, shown in red (B).

\section{E. Layer-by-layer (LbL) assembly}

We use LbL assembly to create PVP/PMAA bilayers, since two polymer layers suffice to get a strong viscoelastic response and more layers would unnecessarily increase the duration of each experiment. Furthermore, the bilayer forms a sufficiently "sharp" interface where a surface excess approach can be expected to work. Ellipsometry thickness measurements reported two polymer layers to have a thickness of about $4 \mathrm{~nm}$ [6].

In order to obtain a reproducible bilayer structure, the following LbL experimental procedure was applied for the radial trough, the DWR and the rising bubble: (i) injection of the PVP solution into the measurement geometry (trough or cup), followed by an equilibration for 1 hour; (ii) exchange of the subphase by inserting acidified Milli-Q water ( $\mathrm{pH} \mathrm{3)}$; (iii) injection of the PMAA solution and equilibration for 1 hour; (iv) exchange of the subphase with Milli-Q water at $\mathrm{pH}$ 3 followed by an equilibration of the assembled system for approximately 15 minutes. Moreover, to ensure a proper exchange of the polymeric solutions from the bulk, for the radial trough, a total volume equal to three times the capacity of the container geometry was exchanged inside the trough (volume radial trough about $300 \mathrm{ml}$ ). In case of the DHR the volume exchanged was equal to about 5 times the cup volume (42 $\mathrm{ml})$. For the rising bubble experiment, the volume exchanged was at least 5 times the container volume $(32 \mathrm{ml})$.

The radial trough used in the present work is based on the design presented in [15], but modified to allow for the exchange of the bulk solutions and the assembly of the two polymer layers at the air-water interface. To this end, a custommade tubing system, made out of PFA (perfluoroalkoxy polymer resin, IDEX Health \& Science, USA) and with an internal diameter of $4 \mathrm{~mm}$, was used. A schematic of the setup is shown in Figure 3. The PFA tubing had a toroidal geometry and was placed on the bottom of the trough, adjacent to the internal wall. Moreover, the tubing system consisted of two sections, internally separated to distinguish between in- and outflow, and each section had twelve evenly spaced holes of 1 $\mathrm{mm}$ on the top side, i.e. the side facing the air-water interface. One section of the tubing system was connected to the inlet of a peristaltic pump (ISM 830, Ismatec, Germany) with PFA tubing of internal diameter of $2 \mathrm{~mm}$, whereas the other section was connected to the outlet of the pump. The configuration of the subphase exchange system as well as the applied flow rate were optimized to guarantee an efficient exchange with independent, yet synchronized, in- and outflow, while reducing the direct impact of the subphase flow itself on the interfacial structure. The peristaltic pump was therefore operated at a flow rate of $15 \mathrm{ml} / \mathrm{min}$.

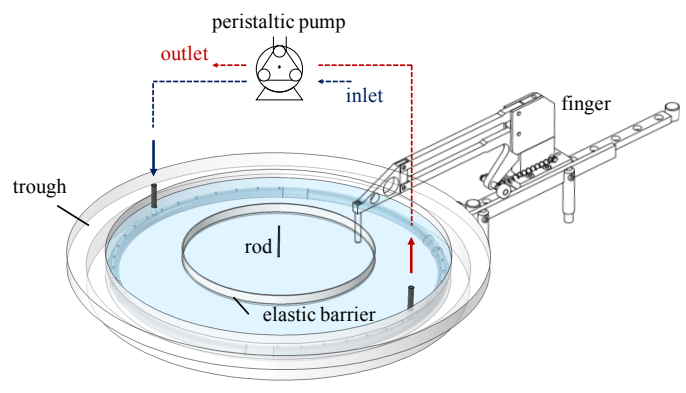

FIG. 3. Schematic of the custom-made subphase exchange system integrated in the radial trough, in which one of the twelve fingers that drive the ribbon is shown.

The assembly of the bilayer system for the shear rheological experiments in the DHR was enabled by using a subphase exchange cell, as described by Schroyen et al. [31]. The inand outlets of the exchange cell were connected to two syringe pumps via an optimized tubing system (Harvard Apparatus, PHD Ultra, USA). The flow rate was kept constant at 6 $\mathrm{ml} / \mathrm{min}$ per in- or outlet. A similar setup was used for the bilayer assembly on the rising bubble, where the syringe pump was connected to the inlet and outlet of the container as shown in Figure 2A. The procedure to assemble the bilayer at the airwater interface followed the same steps as outlined above for the three different setups.

\section{RESULTS AND DISCUSSION}

\section{A. Compressional rheology in a radial trough geometry}

The polymer layers were first investigated separately. In Figure 4, a slow compression-expansion cycle in the radial trough of a PVP (a) and PMAA (b) layer is shown, after adsorption of the polymers at the air-water interface from a diluted bulk solution. The adsorbed, polymeric layers are expected to take on a conformation consisting of "loops" and "trains" [33]. The individual layers are compressible and only a slight increase in surface pressure is observed. This has been attributed to a fast exchange between loops and trains [30]. There is only a small or even negligible hysteresis between compression and expansion, implying that no full desorption of the chains towards the aqueous subphase takes place. At higher compressions, the slight increase in surface concentration and hence in surface pressure suggests we are starting to reach the regime where compression of the "trains" occcurs 
$[6,30]$. Increasing the compression speed did neither change the value of the maximum surface pressure nor the hysteresis, suggesting that we are measuring an intrinsic equilibrium thermodynamic property in the absence of extra stresses.

A
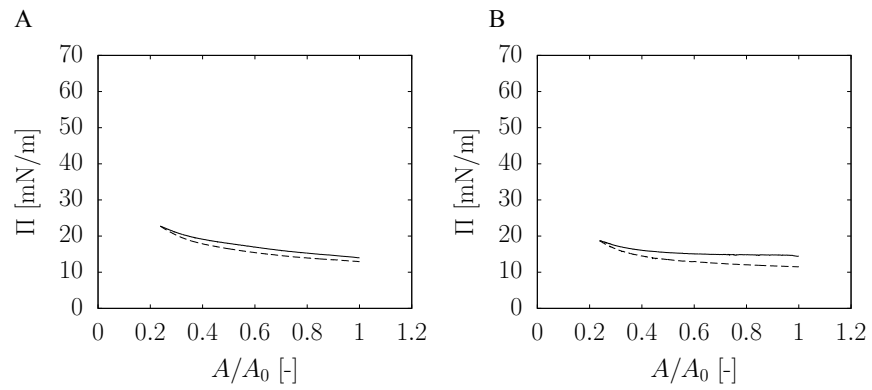

FIG. 4. Surface pressure versus compression ratio during a compression-expansion cycle for a $1 \mathrm{wt} \%$ PVP (A) and PMAA (B) solution at $\mathrm{pH} 3$ in the radial trough. Compression (solid line) and expansion (dashed). Measurements are shown at $1.5 \mathrm{~mm} / \mathrm{min}$ in both cases.

Creating an unstrained reference state for elastic or viscoelastic interfaces is not trivial to achieve experimentally, in particular when the detailed nature of the viscoelastic response is not known a priori. In the present work, this challenge is tackled by using a layer-by-layer assembly technique to form an interface with controlled composition. When the polymers are assembled together at the interface, an effectively insoluble polymeric system is created [30], with enhanced viscoelastic properties. This is clearly visible in the results presented in Figure 5, which shows results from dilatational measurements on the multilayer system. Figure 5A shows a typical step compression experiment in the radial trough of PVP/PMAA bilayers at an air-water interface. The interface is first compressed up to a surface pressure of $20 \mathrm{mN} / \mathrm{m}$, after which the compression is halted and the interface is allowed to equilibrate, resulting in a decrease of surface pressure towards an equilibrium value. Worth mentioning is that this surface pressure is obtained for a compression ratio, $A / A_{0}$ of 0.9 , indicating a more pronounced surface activity compared to the individual polymer layers (Figure 4). Even though the transitions from trains to loops will be hindered by the hydrogen bonding, based on the results of Figure 4, the intrinsic surface tension changes can be expected to be small compared to the stresses occurring in the transient network formed at the interface.

In order to further interrogate the viscoelastic rheological response of such systems when subjected to a uniform deformation, frequency sweeps were performed by small amplitude area oscillations at various surface pressures, which are indicated by the pentagons in Figure 5A. With the goal of probing the rheological response of the system in its initial condition, the first frequency sweep was measured before starting the linear compression as indicated by the blue pentagon in Figure $5 \mathrm{~A}$. The applied area change is $\pm 1 \%$, which was validated to be within the linear response regime (data not shown). In Figure 5B, the resulting dilatational elastic modulus $\left(K^{\prime}\right)$ and dilatational viscous modulus $\left(K^{\prime \prime}\right)$ are plotted against the applied frequency. A dominantly elastic response is obtained even for the unstrained interface (blue squares). Increasing the surface coverage by compression resulted, as expected, in higher values of the dilatational moduli. This response is believed to be predominantly caused by viscoelastic behavior of the polymer network, and for a smaller part by the change in surface energy (which we expect from Figure 4 to be small).

The surface stress tensor can be written as $[5,17]$ :

$$
\boldsymbol{\sigma}^{s}=\sigma(\Gamma, T) \boldsymbol{I}+\boldsymbol{\sigma}^{e}
$$

where $\sigma^{s}$ is the surface stress tensor, $\sigma(\Gamma, T)$ is the state variable surface tension, which depends only on the excess concentration $\Gamma$ and temperature $T, \boldsymbol{I}$ is the surface unit tensor and $\sigma^{e}$ is the surface extra stress tensor, i.e. the "mechanical" stresses that arise due to, e.g. the deformation of an elastic interface. All experiments presented here are performed at constant temperature, and the thermodynamic surface tension of the multilayer is expected to depend only weakly on $\Gamma$, as shown in Figure 4.

In a radial trough, the applied interfacial deformation field is isotropic and homogeneous and the components of the deformation gradient $F_{i j}$ in principal strain space reduce to:

$$
F_{i j}=\left[\begin{array}{cc}
\lambda & 0 \\
0 & \lambda
\end{array}\right]
$$

with $\lambda$ principal stretch. Therefore, in this case, the determinant $J$ of the deformation gradient becomes:

$$
J=\operatorname{det}(\boldsymbol{F})=\lambda^{2}=\frac{A}{A_{0}}
$$

where $A / A_{0}$ represents the change in surface area during the compression. To describe the surface extra stress tensor for large deformations, the material frame indifferent neoHookean model for elastic interfaces, as derived by Pepicelli et al. [15], is used. The model can be written in coordinatefree tensor form as:

$$
\boldsymbol{\sigma}^{e}=\frac{K}{J} \ln (J) \boldsymbol{I}+\frac{G}{J}\left(\tilde{B}^{s}-\frac{1}{2} \operatorname{tr}\left(\tilde{B}^{s}\right) \boldsymbol{I}\right)
$$

where $\tilde{\boldsymbol{B}}^{s}=\boldsymbol{B}^{s} / J$ is the left Cauchy-Green surface strain tensor at constant area, $K$ is the surface compressional/dilational modulus and $G$ is the surface shear modulus. When applying an isotropic deformation, as specified by Equation 2, the following expression for the isotropic part of the extra stress $\sigma_{e}^{\text {iso }}$ is obtained:

$$
\boldsymbol{\sigma}_{e}^{\text {iso }}=K \frac{\ln (J)}{J} \boldsymbol{I}=K \varepsilon \boldsymbol{I}
$$

where $\varepsilon=\ln (J) / J$ represents the Hencky-strain measure. From Equation 5 it follows that there is a linear correlation between the surface stress measured in the radial trough $\left(\sigma_{e}^{\text {iso }}=\Pi \boldsymbol{I}\right)$ and the governing Hencky-strain measure.

Figure 6A shows the surface pressure, relative to the initial value after bilayer assembly $\left(\Pi_{0}=7 \mathrm{mN} / \mathrm{m}\right.$, Figure $\left.5 \mathrm{~A}\right)$, measured in the radial trough (black line) during a step compression. A linear fit is applied (red dashed line) which seems to 
A

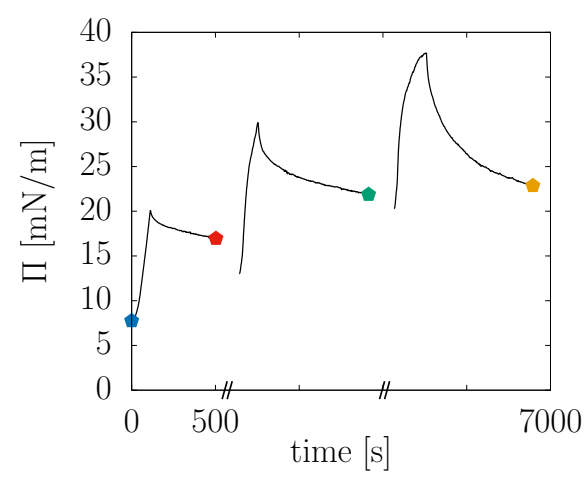

B

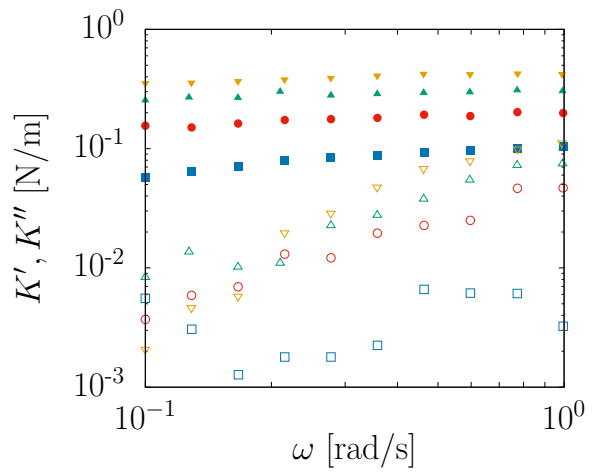

FIG. 5. Behavior of the PVP/PMAA bilayer at an air-water interface in the radial trough. (A) Surface pressure versus time obtained during a sequence of compression-relaxation experiments. The imposed incremental Hencky strains are $-9.6 \%,-10 \%$ and $-20 \%$, respectively (compression speed $=1.5 \mathrm{~mm} / \mathrm{min}$ ). The pentagon symbols indicate the initial surface pressures at which frequency sweeps are performed. (B) Dilatational elastic modulus ( $K^{\prime}$, filled symbols) and dilatational viscous modulus $\left(K^{\prime \prime}\right.$, open symbols) measured by frequency sweeps for increasing surface pressures, corresponding to the different points in diagram A. The applied area change is $\pm 1 \%$.

work well for the strains applied here. From the linear fit, the surface compressional modulus $(K)$ is found to be $168 \mathrm{mN} / \mathrm{m}$. Note that the relaxation time of the bilayers is much larger than the duration of the applied compression, as shown later in this work. Therefore, the Deborah number in this experiment was high, and we can assume that we are mainly probing the purely elastic response of the interface. Figure 6B shows that the values of $K$, obtained from the step compression, and the value of the dynamic dilational modulus, obtained using a frequency sweep in the same initial conditions, are in good agreement.

The value of the compressional modulus is comparable to the value measured for the same system at an oil-water interface in a rising drop apparatus by Le Tirilly and coworkers [6]. In that work, the dilatational modulus was obtained using a pressure measurement, calculating the surface stress at the apex of the droplet where the stresses are expected to be isotropic. In the current work, the rheology of the multi-layer system at the interface between a rising air bubble in water will be further investigated in Section III D.

\section{B. Stress relaxation experiments in a radial trough}

In order to study the relaxation dynamics in more detail, successive compression steps were performed, followed by stress relaxation and a long equilibration time. To describe the viscoelastic behavior of the interface after a step compression, the material properties of the neo-Hookean constitutive model were made time-dependent, i.e. the moduli $K$ and $G$ in Equation 4 are replaced by the compression relaxation modulus $K(t)$ and shear relaxation modulus $G(t)$, respectively. When the strains in the different steps remain in the linear regime and the response is dominated by the extra (rheological) stresses, the principles of linear viscoelasticity, such as the Boltzmann superposition principle [34], should hold. In the present work, this principle can now be evaluated for the first time for interfaces in compression.

In Figure 7, step compression data are presented in terms of relative surface stresses $\left(\Pi-\Pi_{0}, y\right.$-axis) versus time (x-axis). Figure 6(A) shows the same data as in Figure 5 with long waiting times between the experiments as frequency sweeps were carried in between the compression steps, whereas data in Figure 6(B) are from an additional experiment where the Boltzmann superposition principle is being probed more directly, i.e. (a) using shorter waiting times so most of the stress from previous steps has not yet relaxed and (b) a comparison with a a step strain to the same area change in a single step. The strains used in each step are reported in the caption of the figure. Note that in addition to three smaller steps of $-2 \%$ strain, Figure 6(B) also includes the response to a larger step of $-6 \%$ strain. In pure compressional deformation mode, the slope is not affected by the shear relaxation modulus, thus the dilatational relaxation modulus can be calculated directly from the following relation:

$$
K(t)=\frac{\Pi(t)}{\varepsilon}
$$

A linear solid viscoelastic model is fitted to the stress relaxation data, for which the dilatational modulus can be written as:

$$
K(t)=K_{\infty}+K_{1} e^{\frac{-t}{\tau_{d}}}
$$

where $K_{\infty}, K_{1}$ and the relaxation time $\tau_{d}$ are fitting parameters. The value of $K_{\infty}$ represents the purely elastic response, i.e. $\Pi_{\infty}=K_{\infty} \varepsilon$, and determines the value of the surface pressure after relaxation. Equation 6 is fitted to the step compression data, by determining $K_{1}$ using the stress after relaxation, $K_{1}+K_{\infty}$ by the instantaneous jump in stress that results from the step compression, and $\tau_{d}$ by fitting the exponential decay. This procedure yields values of $K_{\infty}=55 \mathrm{mN} / \mathrm{m}, K_{1}=60$ $\mathrm{mN} / \mathrm{m}$ and $\tau_{d}=1008 \mathrm{~s}$ for the data in Figure 7.A. The conditions under which the layer was generated were slightly different in the experiment of Figure 7(B) and values of $K_{\infty}=30$ 
A

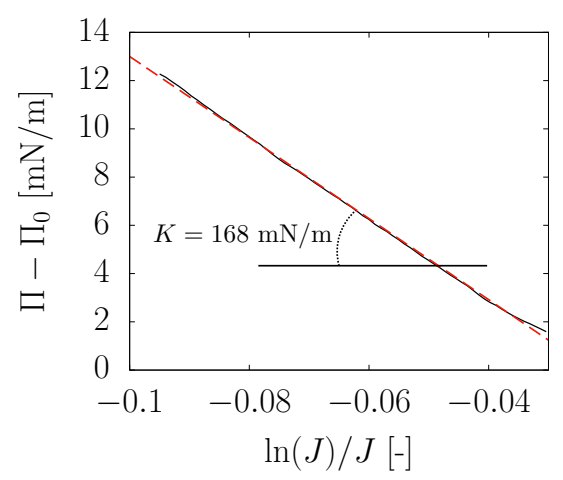

B

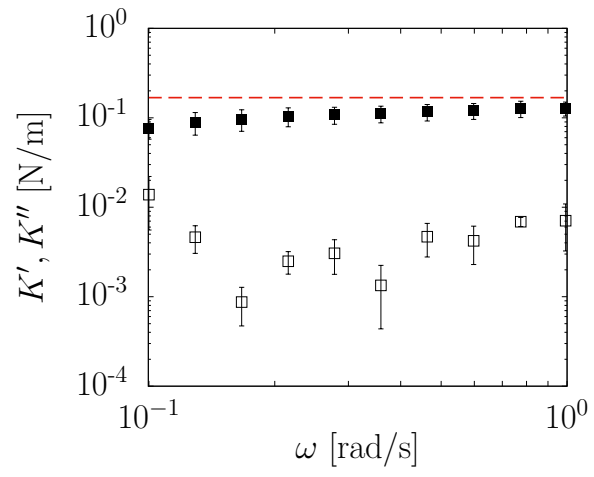

FIG. 6. Radial trough data for a PVP/PMAA bilayer: (A) Relative surface pressure as a function of the Hencky-strain measure $\ln (J) / J$ during the first step compression of Figure 5A (solid black line). A linear fit (dashed red line) to the experimental data yields the surface dilatational modulus (Equation 5). (B) Dilatational frequency sweep performed on a freshly assembled, unstrained interface, applying an area change of $\pm 1 \% . K^{\prime}$ is represented by the filled symbols and $K^{\prime \prime}$ by the empty symbols. The error bars represent the standard deviation of two independent experiments. The red horizontal line represents the value of $K$ calculated from the linear fit of diagram A according to Equation 5.

$\mathrm{mN} / \mathrm{m}, K_{1}=155 \mathrm{mN} / \mathrm{m}$ and $\tau_{d}=140 \mathrm{~s}$ were obrained. The sum of $K_{1}$ and $K_{\infty}$ represents the instantaneous peak value of the dilatational modulus at the end of the compression and is equal to $115 \mathrm{mN} / \mathrm{m}$ for the data in Figure 7(A) and $185 \mathrm{mN} / \mathrm{m}$ for the data in Figure 7(B). These values are comparable to the one calculated in Figure 6 of $168 \mathrm{mN} / \mathrm{m}$. The discrepancy between the measured values for the two interfaces is related to small experimental differences during the creation of the multilayer with respect to spreading and compression history, interface age and potentially small chemical differences (these measurements were carried out at a later time and with the leftovers of a sample batch). A detailed analysis of the microstructural reasons for these subtle dependencies goes beyond the scope of the current work. The goal of the present work is to show that for a given interface, the foundational aspect of linear viscoelastic responses, such as the use of material functions and the Boltzmann superposition principle give good quantitive descriptions.

If the system is linear in its response, the Boltzmann superposition principle (BSP) should be applicable for the extra stresses, and with it, the concept of a "memory function" [34] should be valid. To confirm this, the stress-strain proportionality and the superposition principle must be verified. The relaxation modulus, $K(t)$, can be calculated as in Equation 6, by simply dividing the time-dependent stress $\sigma(t)$ by the chosen strain measure. In addition, according to the principles of proportionality and superposition, the total stress response in case of sequential step-strain experiments performed at times $t_{i}$, is given by:

$$
\sigma(t)=\sum_{i} K\left(t-t_{i}\right) \Delta \varepsilon_{i}=\sum_{i} K\left(t-t_{i}\right) \Delta\left(\frac{\ln (J)}{J}\right)_{i}
$$

where $t_{i}$ represent the times at which each applied step in strain is completed and the relaxation process starts. In principle, in a step strain experiment, the strain should be applied instantaneously; practically, for experimental reasons, the step compression takes a finite time. However, the present case can still be described as a "stress relaxation after a step strain" experiment, because the time scale of the compression step is much shorter than the characteristic relaxation time of the system. In Figure 7, the prediction of the stress evolution obtained from Equation 8 is shown using the values for $K_{1}, K_{\infty}$ and $\tau_{d}$ as mentioned above (red dashed lines) and compared to the experimentally measured stresses (black solid lines), which is a critical test of the BSP in particular for the data in Figure 7(B). Agreement is well within experimental accuracy.

As it can be seen, Equation 8 describes the experimental data presented in Figure 6(A) well for the first two steps and deviates for the last one. When the applied strain remains within the linear viscoelastic regime, the principles of proportionality and superposition are valid, leading to a simple relation between stress and strain, in which the total stress response can be considered as the sum of the responses to the previously applied strains. On the other hand, when the applied strain goes beyond this linear limit, Equation 8 is expected to fail. During the second step, the measured surface stresses already displays a slight overshoot and some indications of non-linearity are observed, although the relaxation dynamics can still be captured well by Equation 8 . For the third step, instead, a lower peak value of stress is measured, together with faster relaxation dynamics, indicative of a strainthinning response, not unexpected for a transient polymer network. This is consistent with a de-structuring of the interface and strain thinning. While for the first two compressions, the incremental step strains were similar and on the order of $10 \%$, for the third case the step strain was $-20 \%$, setting the threshold for linearity in compression below this last value. These observation are confirmed by the data in Figure 6(B), where the step strains applied to the interface where significantly smaller. Both the three smaller steps of $-2 \%$ strain and the larger step of $-6 \%$ strain are described well by Equation 8 , albeit with different values for the material parameters, as mentioned above. It can hence be concluded that as long as the applied strains are within the linear region, the evolution of 
A
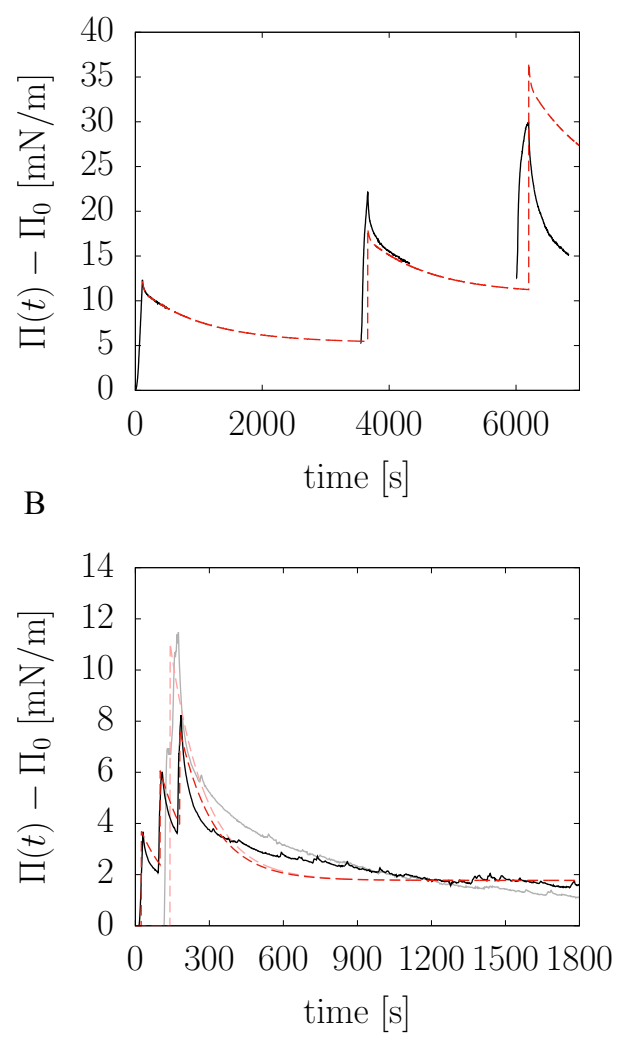

FIG. 7. Boltzmann superposition principle in 2D: Normalized surface pressure versus time for the step compression experiments from Figure 5, where incremental Hencky strains were $-9.6 \%,-10 \%$ and $-20 \%$, for step one, two and three, respectively (A), and a similar experiment on a different multilayer with three incremental Hencky strains of $-2 \%$ (B). Moreover, experimental and modeling results of a large step of $-6 \%$ is also included in (B). In both figures, the dashed red lines are the theoretical predictions and the black solid lines the experimental data. Moreover, in (B), the lighter colored lines indicate the response to a single step strain of $-6 \%$.

the extra viscoelastic stresses in compression can be correctly predicted for any value of applied strain, by simple principles of linear viscoelasticity. Note that despite the relatively large spread on the absolute values of the measured material parameters, we were always able to fit the data using the Boltzmann superposition principle for multiple other interfaces that were created (data not shown).

\section{Shear rheology of the bilayers}

The rheological response of the present system has been investigated also using simple shear deformations. Shear rheology only picks up the deviatoric stresses and hence there is no interplay with potential changes in the thermodynamic contributions. It should be noted that the shear rheological material functions are measured on the interface in its initial conditions after adsorption, since it is not possible to vary the surface coverage after multilayer formation has occured in the subphase exchange cell.

The strain amplitude sweep of Figure 8A provides insight into the structure. The critical strain amplitude for the linear response regime is $\sim 2 \%$. When exiting the linear viscoelastic regime, the viscous modulus $G^{\prime \prime}$ shows a slight increase, which supports the hypothesis of the formation of a multilayer network structure that dissipates energy when breaking up [35]. The presence of a physical network, resulting from the hydrogen-bonded PVP and PMAA, rationalises the frequency response reported in Figure 8B as well. The elastic modulus $G^{\prime}$ is dominant over the entire frequency range and both moduli are weakly dependent on frequency. The evolution of the frequency-dependent shear moduli are in agreement with earlier results for an oil-water interface [6]. In that case, at lower frequencies a crossover point was observed that can be attributed to breaking of temporary network bonds. The contribution to the shear elasticity coming from the individual layers is negligible since for those, a viscous response near the instrument resolution was observed (data not shown). These results confirm that the strong viscoelastic response measured for the bilayer system comes solely from the interlayer interactions.

In order to obtain more insight into the onset of nonlinear behavior, a series of creep-recovery experiments were performed. In Figure 9 the resulting compliance $C(t)$ is shown for increasing applied stresses. For stresses below $0.8 \mathrm{mN} / \mathrm{m}$, the curves overlay into a unique master curve, with $C(t)$ being independent of stress, indicating a linear viscoelastic regime. At higher stresses, the response exits the linear regime with $C(t)$ shifting upwards until finally, at an applied stress of 1.5 $\mathrm{mN} / \mathrm{m}$, the system response becomes non linear. The corresponding total strain at this point equaled $\sim 20 \%$. From this stage on, a constant Newtonian plateau viscosity was observed as a function of stress.

In the linear regime, the viscoelastic response of the system to a creep-recovery experiment can be modelled with simple spring-dashpots models. Although this approach is similar to that applied above, the physical response to dilation or shear is not necessarily the same. For the creep-relaxation experiments in shear, there is a certain measured strain that does not fully recover once the stress is removed, corresponding to a viscous response at long timescales. A generalized KelvinVoigt model is therefore used, which adds an additional viscous term to the model in Equation 7. When assuming a single relaxation process, the compliance $C(t)$ and the recovery compliance $C(t)_{r}$ can be written as follows:

$$
\begin{aligned}
C(t) & =C_{0}+C_{1}\left(1-e^{\frac{-t}{\tau_{s}}}\right)+\frac{t}{\eta_{s, 0}}, t \leq t_{i} \\
C\left(t-t_{i}\right)_{r} & =C\left(t_{i}\right)-\left[C_{0}+C_{1}\left(1-e^{\frac{-\left(t-t_{i}\right)}{\tau_{s}}}\right)\right], t>t_{i}
\end{aligned}
$$

where $C_{0}, C_{1}, \tau_{s}$ and $\eta_{s, 0}$ are fitting parameters and $t_{i}$ the times at which the stresses are released. Similar to the analysis performed for the calculation of the cumulative stress responses in compression, each strain has been fitted starting from the previous end value, as shown in Figure 10. The parameters used for the model predictions are the averaged values 
A

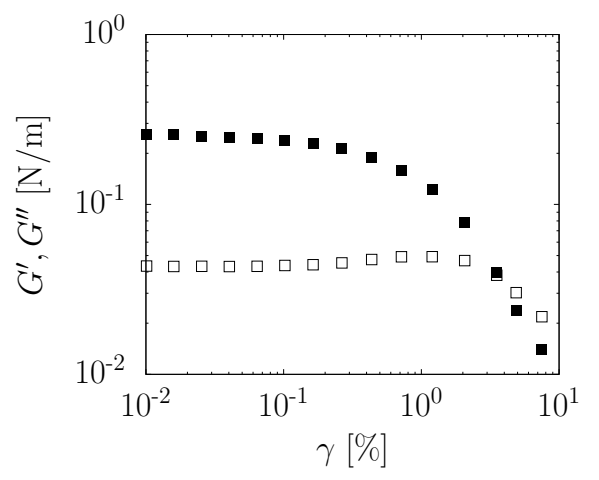

B

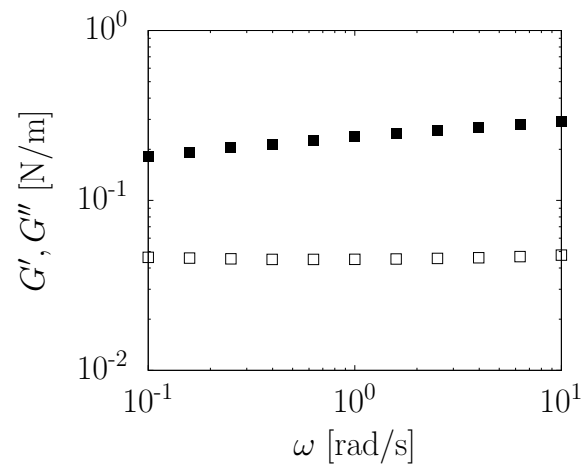

FIG. 8. Interfacial shear rheology results for the PVP/PMAA bilayer. (A) Strain amplitude sweep measured at a frequency of $0.25 \mathrm{rad} / \mathrm{s}$. (B) Frequency sweep performed at $0.1 \%$ strain in the linear viscoelastic regime.

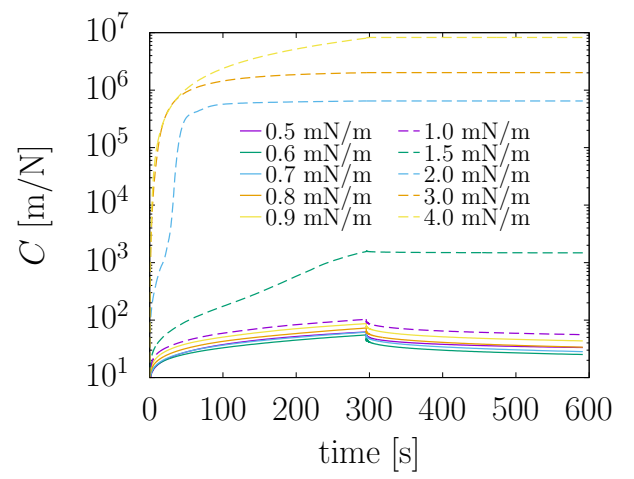

FIG. 9. Creep-recovery compliance for increasing stresses. The multilayer system shows a yielding process for stresses around $1.5 \mathrm{mN} / \mathrm{m}$ and larger.

among the three experiments and are assumed the same for the creep as well as recovery parts. The resulting values are $C_{0}=11.6 \pm 0.77 \mathrm{~m} / \mathrm{N}, C_{1}=12.08 \pm 2.66 \mathrm{~m} / \mathrm{N}$, the dominant shear relaxation time $\tau_{s}=30 \pm 5.40 \mathrm{~s}$ and $\eta_{s, 0}=8 \mathrm{~Pa} \mathrm{~s} \mathrm{~m}$.

Note that the proposed model assumes a single dominant relaxation time and the achievement of a constant strain rate after long enough times, corresponding to a flow regime with a zero shear viscosity. The discrepancies that can be observed between the prediction and the experimental data could arise from the existence of multiple relaxation processes, with an additional relaxation at very long time scales. This explanation would also agree with a) the occurrence of a lowfrequency crossover point in oscillatory shear experiments and b) the longer relaxation dynamics detected by step compression measurements in the radial trough $\left(\tau_{d}\right)$.

\section{Rising bubble experiments}

In this section, we use a rising bubble setup to evaluate the stresses in the PVP/PMAA bilayer for large compressions. A standard procedure is to fit the unstrained shape of the bub-

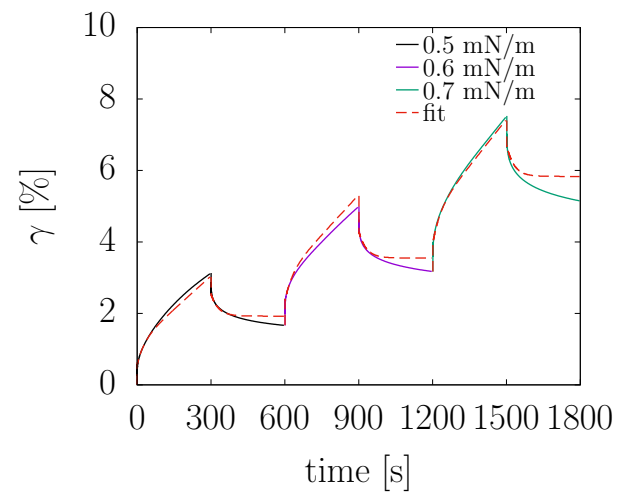

FIG. 10. Theoretical prediction of the creep-relaxation behavior within the linear viscoelastic regime. The generalized Kelvin Voigt model prediction is shown in dotted lines, the experimental data as full lines.

ble with the Young-Laplace equation, which yields the surface tension and the pressure inside the bubble, using the underlying assumption that the surface stresses are uniform and isotropic. When applying a deformation in a rising bubble experiment, the deformation of the surface at the apex of the bubble must be isotropic due to symmetry considerations. However, near the capillary the bubble is constrained in the radial direction, but not in the meridional direction ( $r$ and $s$ direction in Figure 11, respectively), which results in a deformation of the bubble surface that is non-uniform and anisotropic. If surface stresses are directly coupled to surface deformation, such as is done in the neo-Hookean model as given in Equation 4, this yields non-uniform and anisotropic surface stresses, rendering the application of the Young-Laplace equation erroneous [16, 36-39].

We start by making a bubble in water with a volume of $V_{0}=12 \mu \mathrm{L}$, followed by an exchange for introducing the PVP solution. The progression of the calculated surface tension and calculated/measured pressures are shown in Figure 12A and B. Note that the first 1000 seconds of the adsorption curve for PVP are not shown due to problems with the image acqui- 


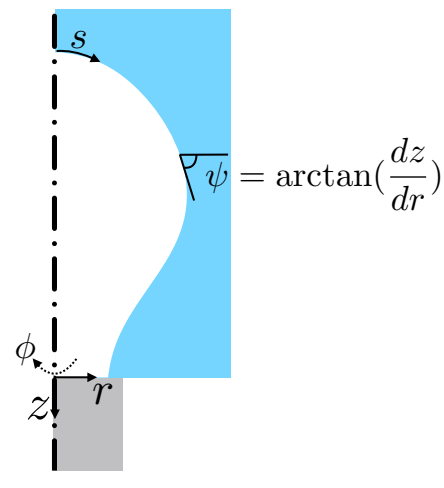

FIG. 11. Definitions of the coordinates as used for the rising bubble experiment.

sition. It can be observed that the surface tension drops only slightly during the adsorption, and that the measured pressure corresponds well to the calculated pressure, indicating that the surface stresses are uniform and isotropic. This confirms the low surface activity of PVP by itself (see Figure 4), although the decrease in surface tension for the rising bubble, where the air-water interface is curved, is about $10 \mathrm{mN} / \mathrm{m}$ lower than the one reported for a planar interface in Figure 4A. The Worthington number (Wo) was calculated to be at least 0.6 , which is high enough for accurate measurement using standard tensiometry $[16,40]$.

After the adsorption of PVP, we exchange the subphase with acidified water and subsequently introduce the PMAA solution. The evolution of the surface tension and pressure are shown in Figure 12C and D. Again, an additional small drop in the measured surface tension can be observed. Moreover, oscillations can now be seen in the measured pressure signal. Upon closer inspection, the oscillations correspond to oscillations of the volume of the drop, which oscillates mildly around the set-point. Even though the resulting surface strains are small (i.e. less then 1\%), the effect on the measured pressure is on the order $10 \mathrm{~Pa}$, which indicates the formation of a highly elastic network at the interface. It is interesting to note that the measured pressure is able to pick up even such small strains, whereas the pressure estimated by the Young-Laplace fit does not. Finally, we exchange the PMAA solution for water and perform compression experiments by reducing the volume of the drop rapidly by steps of $1 \mu \mathrm{L}$, and each time letting the interface relax for at least 1200 seconds.

The resulting surface tension and pressures are shown in Figure 12E and F. Reductions of the calculated surface tension on the order of $10 \mathrm{mN} / \mathrm{m}$ for each step compression are observed, which shows a slight relaxation after each step strain. A similar trend is observed in the calculated Young-Laplace (YL) pressure, which shows that the predicted pressure in the bubble decreases roughly linearly with decreasing surface area. However, the measured pressure shows a different response, and discrepancies with the YL pressure become significant, which grow for each step strain, indicating that we are in a regime where the extra stresses can no longer be neglected. Moreover, the relaxation of the surface stresses is more clearly visible in the measured pressure, with a relaxation time that is similar to the one that was observed in previous sections, see e.g. Figure 5. Note that the strong dips in the measured pressure occur during the step compression, and are most likely related to flow inside the tubing system.
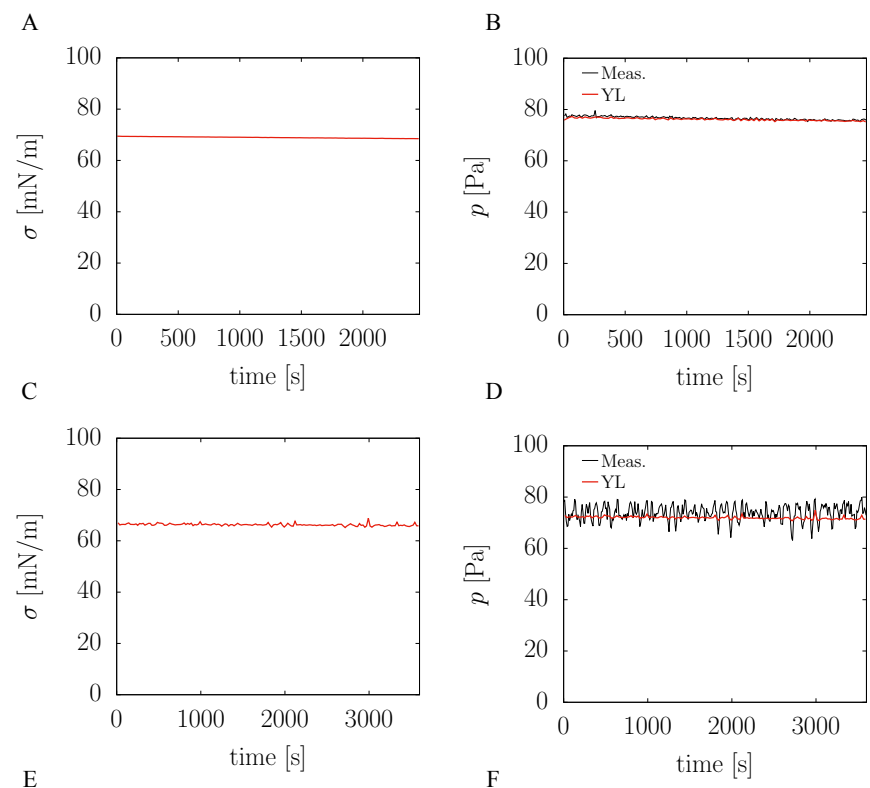

E
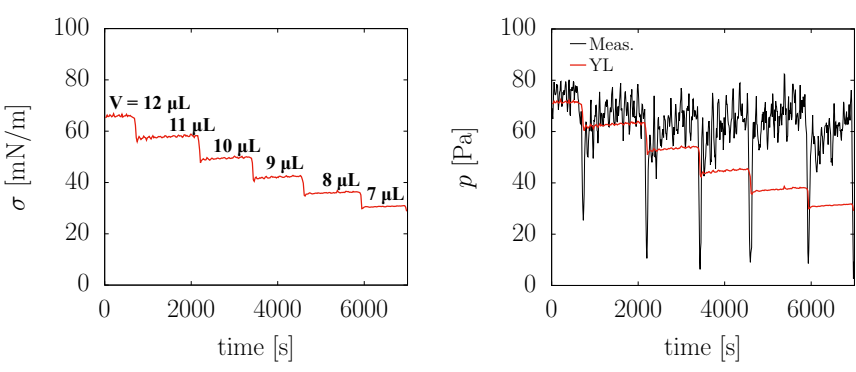

FIG. 12. Comparison between the YL pressure and the measured pressure. Left: the calculated surface tension using the YoungLaplace approximation during the adsorption of PVP (A), the subsequent adsorption of PMAA (C) and the compression experiments (E). Right: the calculated YL pressure and the measured pressure during the adsorption of PVP (B), the subsequent adsorption of PMAA (D) and the compression experiments $(\mathrm{F})$.

In the rising bubble experiment, the surface deformation cannot be imposed, but is rather an outcome of an imposed step in the bubble volume. In Figure 13 we show images of the bubbles at the end of each compression step, and report the compression ratio between the initial surface area of the bubble (which is $A_{0}=24.8 \mathrm{~mm}^{2}$ ) and the surface area after the compression. In the same figure, we also report the compressive surface strain, using the definition $\varepsilon=\ln (J) / J$.

It is clear from Figure $12 \mathrm{~F}$ that the Young-Laplace approximation yields results that are inconsistent with the measured values of the pressure. As an alternative approach, the capillary meniscus dynanometry (CMD) method was developed by Danov et al. [37]. Using the CMD method, the stresses in the interface can be determined directly from a force balance, using the measured pressure and bubble shape through 


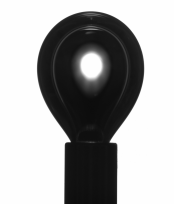

$A / A_{0}=0.95$ $\epsilon=-5 \%$

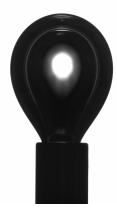

0.89

$-13 \%$

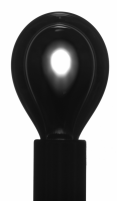

0.83

$-22 \%$

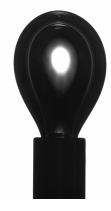

0.77

$-34 \%$

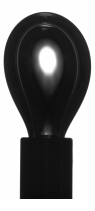

0.73

$-43 \%$
FIG. 13. Bubble shapes at the end of each compression step. The values of $A / A_{0}$ represent the ratio of the surface area and the initial surface area, whereas $\varepsilon$ denotes the compressive surface strain, defined by $\varepsilon=\ln (J) / J$.

the following relations $[16,37]$ :

$$
\begin{aligned}
\sigma_{s} & =\frac{1}{2 r \sin (\psi)}\left(p r^{2}+g \rho \int_{0}^{s} r^{2} \sin (\psi) d s-g \rho z r^{2}\right) \\
\sigma_{\phi} & =\frac{p-\rho g z-\sigma_{s} \kappa_{s}}{\kappa_{\phi}}
\end{aligned}
$$

where $\kappa_{\phi}$ and $\sigma_{\phi}$ are the principal curvature and stress in circumferential direction, respectively, and $\kappa_{s}$ and $\sigma_{s}$ are the principal curvature and stress in meridional direction, $p$ is the pressure difference across the interface at $z=0, \rho$ is the density difference, $g$ is the gravitational acceleration and $z$ is the vertical coordinate, as defined in Figure 11.

The contour of the interface is approximated by a Chebyshev expansion, hence differentiation and integration can be easily performed to determine the curvatures $[16,41]$. However, the experimental data must be differentiated in order to obtain the curvatures, amplifying unavoidable noise. This problem exists both for determining $\sigma_{s}$ and $\sigma_{\phi}$, although it is worse for the latter [16]. One approach to tackle this problem is to smooth the experimental data prior to taking the derivatives. However, this could induce unwanted curvature, influencing the obtained stresses considerably. The approach taken here is to inspect the curvature obtained from a large range of $N$, which is the number of Chebyshev base functions used in the approximation. Note, that near the capillary, the curvature tends to oscillate considerably with $N$, which is most likely due to lack of boundary conditions on the second derivative of the shape, which is inherent to the problem. We therefore excluded the last $5 \%$ of the curve.

The results of applying the CMD method to the compressed bubbles are shown in Figure 14. The calculations are performed at the end of each relaxation period, i.e. the stresses are "true" elastic stresses that will not relax anymore. The results indicate that $\sigma_{s}$ is independent of $N$, whereas $\sigma_{\phi}$, which needs a higher order derivative, is only weakly dependent on $N$. We can therefore be confident that these plots describe the actual stresses in the interface. It is clear that with increasing compression, the stresses become more anistropic and nonuniform. Moreover, the Young-Laplace prediction of the surface tension, which is included in the graphs, shows that the error made using that approach is large, and increases with compression. It should be noted that the extent by which the stresses are non-uniform depends on the constitutive properties of the interface but also on the size of the bubble, relative to the capillary radius. Therefore, when solving the inverse problem, i.e. going from the drop shape to the surface tension and stress as usually done, the error made in estimating the surface tension from the drop shape alone will depend on the extent of drop deformation. For smaller bubbles the contribution of the surface tension will be more important and the role of extra stresses will be smaller for a given strain, which may explain the agreement between the YL pressure and measured pressure reported previously [30]. But at a certain bubble size, the change in curvature along the drop becomes too small $(W o<0.3)$ for the YL fit to be accurate. In general, YL fits are not sufficiently sensitive to evaluate the effect of surface stresses [16]. This problem could be tackled by solving an inverse problem that takes anisotropy into account, e.g. using the stress fitting elastometry method [16]. It was shown that this can yield accurate predictions for $K$, but less so for $G$ [16]. In the present work, this was not done since the values of $K$ and $G$ were already obtained from experiments in different geometries that impose well-defined deformations.

To conclude this section, we compare the experimentally obtained surface stresses as determined by the CMD method and given in Figure 14, to the stresses that are predicted by the neo-Hookean constitutive model (Equation 4). For the axisymmetric rising bubble, the surface stresses, including the isotropic thermodynamic surface tension $\sigma$, can be written in principal strain space as [15]:

$$
\begin{gathered}
\sigma_{s}-\sigma=\frac{K}{J} \ln (J)+\frac{G}{2}\left(\frac{1}{\lambda_{\phi}^{2}}+\frac{1}{\lambda_{s}^{2}}\right) \\
\sigma_{\phi}-\sigma=\frac{K}{J} \ln (J)-\frac{G}{2}\left(\frac{1}{\lambda_{\phi}^{2}}+\frac{1}{\lambda_{s}^{2}}\right)
\end{gathered}
$$

where $\lambda_{\phi}$ and $\lambda_{s}$ are the stretches in circumferential and meridional direction, respectively, and $J=\lambda_{\phi} \lambda_{s}$ is the change in area. Using the spectral method as presented by Nagel et al. [16], we apply the neo-Hookean model to predict the surface stresses $\sigma_{s}$ and $\sigma_{\phi}$ for the given surface compressions $A / A_{0}=0.95,0.89,0.83$ and 0.77 , at the end of each compression step. The model needs values for $\sigma, K$ and $G$ as input. The thermodynamic contribution to the surface stress, $\sigma$, will be assumed constant, which is justified by the individual components' weak dependency of the surface tension on the compression of the interface, as was shown in Figure 4. For $\sigma$, we use $66 \mathrm{mN} / \mathrm{m}$, which corresponds to the surface tension of the PVP/PMAA multi-later before compression, as shown in Figure 12E. For the surface dilatational modulus we use the value obtained in the radial trough for relaxation after a step compression (see Section III B), since the stresses shown in Figure 14 are determined at the end of the relaxation period, i.e. $K=K_{\infty}=55 \mathrm{mN} / \mathrm{m}$. Finally, the value of the shear modulus used in the model is based on the creeprecovery experiments as shown in Section III C. A shear modulus can be determined by the inverse of the compliance values, i.e. $1 / C_{0} \approx 80 \mathrm{mN} / \mathrm{m}$. However, as can be seen from the fit in Figure 10, the total recovery, or compliance, is underestimated by the fit. We therefore use a value $G=40$ 

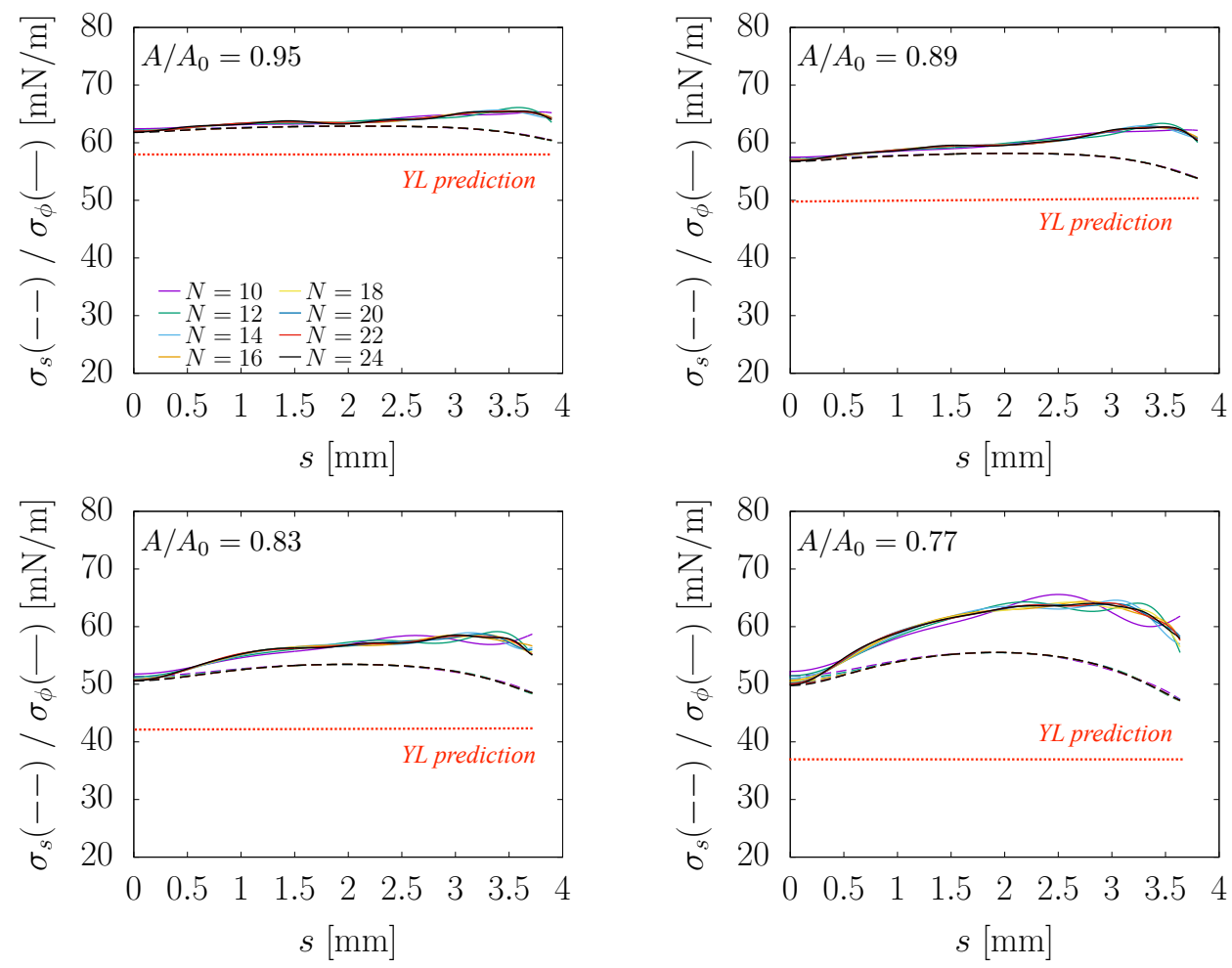

FIG. 14. Stresses in the interface as determined by the CMD method, at the end of each compression step. Dashed lines represent the stress in meridional direction and solid lines the stress in circumferential direction. The dotted lines represent the Young-Laplace prediction for the surface tension. $N$ represents the number of Chebyshev base functions used in the numerical representation of the interface.

$\mathrm{mN} / \mathrm{m}$, which was found to describe the stresses well, especially with regard to the anisotropy of the surface stresses. Note, that these values of $K$ and $G$ can also be written in terms of a surface Young's modulus $E$ and a surface Poisson ration $v$, which yields $E=4 G K /(G+K) \approx 93 \mathrm{mN} / \mathrm{m}$ and $v=(K-G) /(K+G) \approx 0.16[16]$.

The model results are shown in Figure 15, where also the stresses as measured in the experiment using $N=24$ are shown for comparison. For relatively small surface compressions, the model predicts both the level of the stresses and the anistropy well. Significant deviations start to occur for $A / A_{0}=0.83$, and the model fails to predict the stresses for $A / A_{0}=0.77$. As mentioned before, the last $5 \%$ of the measured stress is excluded from the analysis, which explains the difference in length between the measured and calculated stress curves, i.e. the maximum value of $s$. The corresponding values for $\varepsilon=\ln (J) / J$ were given in Figure 13. In Figure 16 we report the calculated local strains for the rising bubble experiment. As expected, the circumferential strain $\lambda_{\phi}$ and meridional strain $\lambda_{s}$ are equal near the apex of the $\operatorname{drop}(s=0)$, yielding an isotropic deformation and thus an isotropic stress state. However, the interface cannot deform circumferentially near the neck region due to the presence of the needle. This is clearly visible in the plot, with the value of $\lambda_{\phi}$ going to one at the needle.

Finally, to investigate the transient behavior of the model as well, we performed calculations using the spectral method, but with a time-dependent value of $K$. The expression used for $K(t)$ is similar to Equation 7, with values for $K_{1}, K_{\infty}$ and $\tau$ similar to the ones found in the radial trough measurement, i.e. $K_{\infty}=55 \mathrm{mN} / \mathrm{m}, K_{1}=60 \mathrm{mN} / \mathrm{m}$ and $\tau=1008$ seconds. The value of $G=40 \mathrm{mN} / \mathrm{m}$ is kept constant since its influence on the capillary pressure, which is the quantity we will investigate here, is small. The first three step strain experiments are simulated by calculating the stresses after each individual step strain, using the state right before the step as the reference state, and using the time dependent $K(t)$ for each process. The surface stress is then assumed to be the sum of the stresses resulting from the individual step strains, i.e. applying Boltzmann superposition principle. The predicted pressure, together with the measured pressure is reported in Figure 17, which shows remarkable agreement, even for absolute surface strains up to $22 \%$. As mentioned before, we note that the strong dips in the measured pressure occur during the step compression, and are most likely related to flow inside the tubing system. Overall, we can conclude that the neo-Hookean constitutive model is able to predict the surface stresses and capillary pressure well, also for a mixed deformation, such as the one occurring in the rising bubble. The model predictions are reasonable up to a strain of around $20 \%$, which is consistent with earlier results on the interfacial neo-Hookean model for large deformations [15]. 

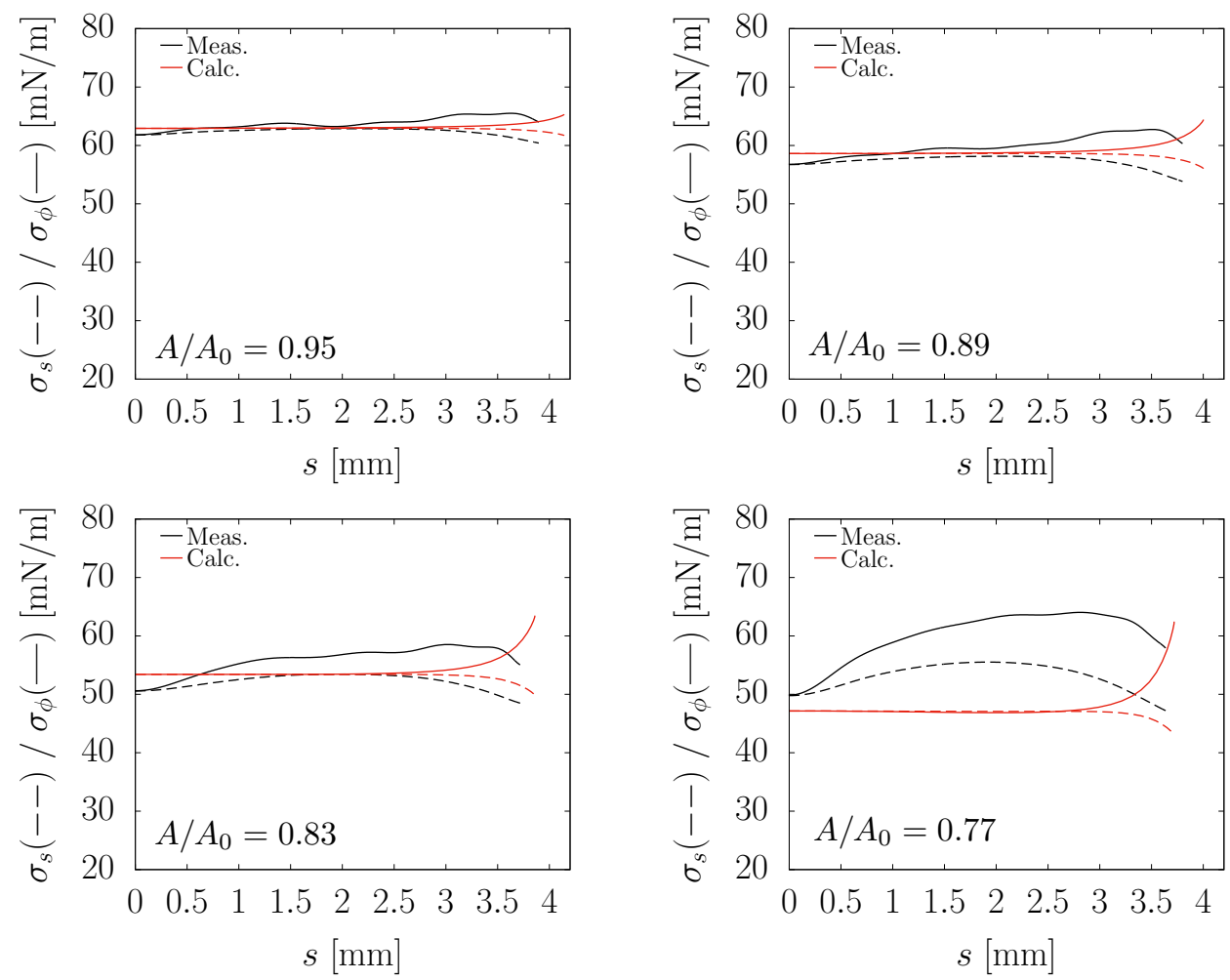

FIG. 15. The calculated surface stresses (red) as predicted by the neo-Hookean constitutive model, using values of $\sigma=66 \mathrm{mN} / \mathrm{m}, K=55$ $\mathrm{mN} / \mathrm{m}, G=40 \mathrm{mN} / \mathrm{m}$. For comparison, the measured values (black), determined by CMD, from Figure 14 are also shown (for $N=24$ ). Dashed lines represent the stress in meridional direction and solid lines the stress in circumferential direction.

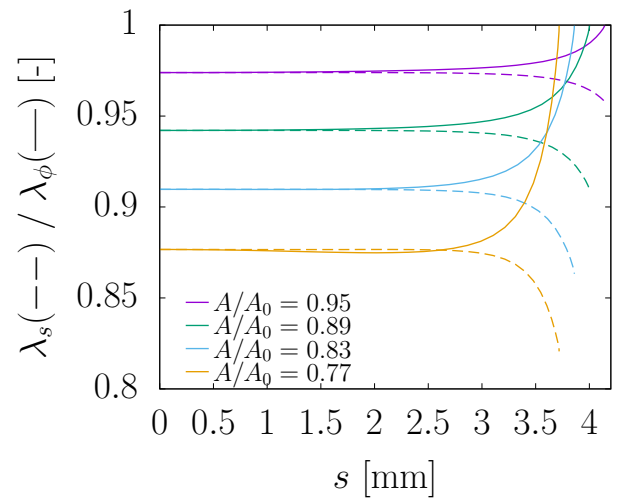

FIG. 16. The calculated strains as predicted by the neo-Hookean constitutive model, using the same values for the material parameters as in Figure 15.

\section{CONCLUSIONS}

In this work, a transient polymer bilayer, assembled at the water-air interface, has been used as a model viscoelastic system for studying the effects of surface viscoelasticity and nonlinear behavior in shear and, for the first time, in uniform compression under rheometric conditions. Moreover, the same system was studied in a more complex deformation in a rising bubble experiment. The material functions were deter- mined experimentally in well-defined kinematic conditions using compression in a radial trough, and in simple shear using a double wall ring geometry.

The experiments performed in the radial trough made it possible to distinguish the different contributions to the overall viscoelastic response measured in compression. The compressibility could be neglected compared to the viscoelastic effects arising due to a transient network. Moreover, a typical viscoelastic solid-like response was detected during step compression experiments. The material functions were identified in the linear response regime, and the Boltzmann superposition principle was shown to apply to the evolution of the extra viscoelastic stresses for any given strain history.

Similar results were obtained for simple shear deformations. The frequency-dependent material functions, $G^{\prime}$ and $G^{\prime \prime}$, are consistent with the presence of a viscoelastic network with a limited linear response regime. In the linear viscoelastic regime, a generalized Kelvin-Voigt model was used to calculate the constitutive parameters for this system under a creep deformation.

The rising bubble experiments confirm the existence of a strong viscoelastic network. It is shown that standard tensiometry fails for these type of systems, and alternative approaches, such as the stress fitting elastrometry must be applied. Moreover, it is shown that the neo-Hookean constitutive model performs well up to moderate strains, also for the complex deformations that arise in a deforming bubble. To go beyond moderate deformations, more refined constitutive models are 
required.

In conclusion, these results confirmed that, both in shear and dilatation, the viscoelastic response measured in these supramolecular polymer layers is dominated by strong interlayer interactions.

\section{ACKNOWLEDGMENTS}

The authors thank Aster Rabinovitch and Gustavo Alonso Barrientos Sandoval for help with the rising bubble experiments. NOJ and JV gratefully acknowledge TOTAL S.A. for financial support. CT and CM gratefully acknowledge ANR JCJC INTERPOL financial support.

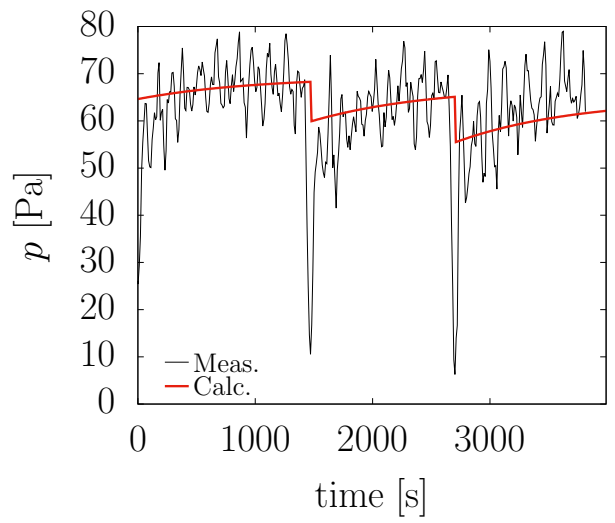

FIG. 17. Measured (black) and predicted (red) transient pressure assuming a constant surface tension and a quasi linear neo-Hookean surface response, during the step strain experiments in the rising bubble. Time has been shifted such that 0 corresponds to the end of the first step strain.
[1] D. Langevin. Influence of interfacial rheology on foam and emulsion properties. Advances in Colloid and Interface Science, 88(1-2):209-222, 2000.

[2] D. Guzey and D.J. McClements. Formation, stability and properties of multilayer emulsions for application in the food industry. Advances in Colloid and Interface Science, 128-130:227248, 2006.

[3] D.O. Grigoriev, T. Bukreeva, H. Möhwald, and D.G. Shchukin. New method for fabrication of loaded micro- and nanocontainers: Emulsion encapsulation by polyelectrolyte layer-bylayer deposition on the liquid core. Langmuir, 24(3):999-1004, 2008.

[4] G. G. Fuller and J. Vermant. Complex fluid-fluid interfaces: Rheology and structure. Annual Review of Chemical and Biomolecular Engineering, 3(1):519-543, 2012.

[5] E. Hermans, M. Saad Bhamla, P. Kao, G. G. Fuller, and J. Vermant. Lung surfactants and different contributions to thin film stability. Soft Matter, 11(41):8048-8057, 2015.

[6] S. Le Tirilly, C. Tregouët, S. Bone, C. Geffroy, G. Fuller, N. Pantoustier, and C. Perrin, P. Monteux. Interplay of hydrogen bonding and hydrophobic interactions to control the mechanical properties of polymer multilayers at the oil-water interface. ACS Macro Letters, 4(1):25-29, 2015.

[7] P. Erni. Deformation modes of complex fluid interfaces. Soft Matter, 7(17):7586, 2011.

[8] T. Verwijlen, L. Imperiali, and J. Vermant. Separating viscoelastic and compressibility contributions in pressure-area isotherm measurements. Advances in Colloid and Interface Science, 206:428-436, 2014.

[9] S. Reynaert, C.F. Brooks, P. Moldenaers, J. Vermant, and G.G. Fuller. Analysis of the magnetic rod interfacial stress rheometer. Journal of Rheology, 52(1):261-285, 2008.

[10] P. Erni, P. Fischer, E.J. Windhab, V. Kusnezov, H. Stettin, and J. Läuger. Stress- and strain-controlled measurements of interfacial shear viscosity and viscoelasticity at liquid/liquid and gas/liquid interfaces. Review of Scientific Instruments, 74(11):4916-4924, 2003.
[11] S. Vandebril, A. Franck, G.G. Fuller, P. Moldenaers, and J. Vermant. A double wall-ring geometry for interfacial shear rheometry. Rheologica Acta, 49(2):131-144, 2010.

[12] S. Fitzgibbon, E.S.G. Shaqfeh, G.G. Fuller, and T.W. Walker. Scaling analysis and mathematical theory of the interfacial stress rheometer. Journal of Rheology, 58(4):999-1038, 2014.

[13] O.H. Soo-Gun and J.C. Slattery. Disk and biconical interfacial viscometers. Journal of Colloid And Interface Science, 67(3):516-525, 1978.

[14] E. Aumaitre, D. Vella, and P. Cicuta. On the measurement of the surface pressure in langmuir films with finite shear elasticity. Soft Matter, 7(6):2530-2537, 2011.

[15] M. Pepicelli, T. Verwijlen, T. A. Tervoort, and J. Vermant. Characterization and modelling of langmuir interfaces with finite elasticity. Soft Matter, 13:5977-5990, 2017.

[16] M. Nagel, T. Tervoort, and J. Vermant. From drop-shape analysis to stress-fitting elastometry. Advances in Colloid and Interface Science, 247:33-51, 2017.

[17] N.O. Jaensson and J. Vermant. Tensiometry and rheology of complex interfaces. Current Opinion in Colloid \& Interface Science, 37:136-150, 2018.

[18] Z.A. Zell, A. Nowbahar, V. Mansard, L.G. Leal, S.S. Deshmukh, J.M. Mecca, C.J. Tucker, and T.M. Squires. Surface shear inviscidity of soluble surfactants. Proceedings of the National Academy of Sciences, 111(10):3677-3682, 2014.

[19] C.F. Brooks, G.G. Fuller, C.W. Frank, and C.R. Robertson. An Interfacial Stress Rheometer To Study Rheological Transitions in Monolayers at the Air-Water Interface. Langmuir, 15(7):2450-2459, 1999.

[20] T. Verwijlen, P. Moldenaers, and J. Vermant. A fixture for interfacial dilatational rheometry using a rotational rheometer. The European Physical Journal Special Topics, 222(1):83-97, 2013.

[21] K.H. Kim, S.Q. Choi, J.A. Zasadzinski, and T.M. Squires. Interfacial microrheology of dppc monolayers at the air-water interface. Soft Matter, 7(17):7782-7789, 2011.

[22] E. Hermans and J. Vermant. Interfacial shear rheology of dppc under physiologically relevant conditions. Soft Matter, 
10(1):175-186, 2014.

[23] K.H. Kim, S.Q. Choi, J.A. Zasadzinski, and T.M Squires. Nonlinear chiral rheology of phospholipid monolayers. Soft matter, 14(13):2476-2483, 2018.

[24] S. Reynaert, P. Moldenaers, and J. Vermant. Interfacial rheology of stable and weakly aggregated two-dimensional suspensions. Physical Chemistry Chemical Physics, 9(48):6463-6475, 2007.

[25] B. Madivala, J. Fransaer, and J. Vermant. Self-assembly and rheology of ellipsoidal particles at interfaces. Langmuir, 25(5):2718-2728, 2009.

[26] J.H.J. Thijssen and J. Vermant. Interfacial rheology of model particles at liquid interfaces and its relation to (bicontinuous) pickering emulsions. Journal of Physics: Condensed Matter, 30(2):023002, 2017.

[27] A.J. Mendoza, E. Guzmán, F. Martínez-Pedrero, H. Ritacco, R.G. Rubio, F. Ortega, V.M. Starov, and R. Miller. Particle laden fluid interfaces: Dynamics and interfacial rheology. Advances in Colloid and Interface Science, 206:303-319, 2014.

[28] G.M. Radulova, K. Golemanov, K.D. Danov, P.A. Kralchevsky, S.D. Stoyanov, L.N. Arnaudov, T.B.J. Blijdenstein, E.G. Pelan, and A. Lips. Surface Shear Rheology of Adsorption Layers from the Protein HFBII Hydrophobin: Effect of Added $\beta$ Casein. Langmuir, 28(9):4168-4177, 2012.

[29] J. Burke, A. Cox, J. Petkov, and B.S. Murray. Interfacial rheology and stability of air bubbles stabilized by mixtures of hydrophobin and $\beta$-casein. Food Hydrocolloids, 34:119-127, 2014.

[30] S. Le Tirilly, C. Tregouët, M. Reyssat, S. Bône, C. Geffroy, G.G. Fuller, N. Pantoustier, P. Perrin, and C. Monteux. Interfacial Rheology of Hydrogen-Bonded Polymer Multilayers Assembled at Liquid Interfaces: Influence of Anchoring Energy and Hydrophobic Interactions. Langmuir, 32(24):6089-6096, 2016.

[31] B. Schroyen, D.Z. Gunes, and J. Vermant. A versatile subphase exchange cell for interfacial shear rheology. Rheologica Acta, 56:1-10, 2016.
[32] F. Peters and D. Arabali. Interfacial tension between oil and water measured with a modified contour method. Colloids and Surfaces A: Physicochemical and Engineering Aspects, 426:15, 2013.

[33] C. Barentin and J. Joanny. Surface pressure of adsorbed polymer layers. Effect of sticking chain ends. Langmuir, 10:18021811, 1999.

[34] J.D. Ferry. Viscoelastic properties of polymers. 3rd Edition, 1980.

[35] J. Mewis and N. J. Wagner. Colloidal suspension rheology. Cambridge University Press, 2011.

[36] N.A. Alexandrov, K.G. Marinova, T. Gurkov, K.D. Danov, P.A. Kralchevsky, S.D. Stoyanov, T.B.J. Blijdenstein, and L.N. Arnaudov. Interfacial layers from the protein hfbii hydrophobin: Dynamic surface tension, dilatational elasticity and relaxation times. Journal of Colloid and Interface Science, 376:296-306, 2012.

[37] K.D Danov, R.D. Stanimirova, P.A. Kralchevsky, K.G. Marinova, N.A. Alexandrov, S.D. Stoyanov, T.B.J. Blijdenstein, and E.G. Pelan. Capillary meniscus dynamometry-method for determining the surface tension of drops and bubbles with isotropic and anisotropic surface stress distributions. Journal of Colloid and Interface Science, 440:168-178, 2015.

[38] J.K. Ferri, P.A.L. Fernandes, J.T. McRuiz, and F. Gambinossi. Elastic nanomembrane metrology at fluid-fluid interfaces using axisymmetric drop shape analysis with anisotropic surface tensions: deviations from Young-Laplace equation. Soft Matter, 8(40):10352, 2012.

[39] V. Pauchard, J.P. Rane, and S. Banerjee. Asphalteneladen interfaces form soft glassy layers in contraction experiments: A mechanism for coalescence blocking. Langmuir, 30(43):12795-12803, 2014.

[40] J.D. Berry, M.J. Neeson, R.R. Dagastine, D.Y.C. Chan, and R.F. Tabor. Measurement of surface and interfacial tension using pendant drop tensiometry. Journal of Colloid and Interface Science, 454:226-237, 2015.

[41] L.N. Trefethen. Spectral methods in MATLAB, volume 10. Siam, 2000. 